Cómo citar este trabajo: Cordera, R., Nogués, S., \& González-González, E. (2019). El reto de incorporar indicadores en los sistemas de evaluación y seguimiento de los Planes Regionales de Ordenación Territorial en España. Boletín de la Asociación de Geógrafos Españoles, 81, 2726, 1-32. http://dx.doi.org/10.21138/bage.2726

\title{
El reto de incorporar indicadores \\ en los sistemas de evaluación y seguimiento de los Planes Regionales de Ordenación Territorial en España
}

The challenge of introducing indicators in the evaluation
and monitoring systems of Spanish Regional Plans

Rubén Cordera

corderar@unican.es

Soledad Nogués

soledad.nogues@unican.es

\section{Esther González-González}

gonzalezme@unican.es

Grupo GEURBAN

Universidad de Cantabria (España)

\section{Resumen}

El progresivo proceso de europeización de la planificación territorial ha promovido la aparición de nuevos objetivos y mecanismos de control de los planes de ordenación, como son el desarrollo sostenible y los sistemas de evaluación y seguimiento. Sin embargo, en España, a diferencia de otros países que cuentan con directrices o guías nacionales, esta inclusión no se ha realizado de manera sistemática. Este artículo tiene como objetivo comprobar hasta qué punto y cómo se está integrando la evaluación y seguimiento de los planes territoriales españoles prestando especial atención al uso de sistemas de indicadores. La revisión de los planes actuales permite afirmar que 
España está todavía dando sus primeros pasos, dado que, de las 17 comunidades autónomas, diez contemplan sistemas de evaluación y seguimiento específicos, mediante la elaboración de planes o informes, y solo cinco los han desarrollado e implementado incluyendo el uso de sistemas de indicadores. Además, las metodologías empleadas difieren significativamente en cuanto a qué debe medirse, es decir, en cómo se conceptualiza la sostenibilidad, y al número y tipo de indicadores seleccionados. De cara al futuro se recomienda tender a una mayor convergencia en la definición de las metodologías de evaluación y seguimiento que contemplen el uso de indicadores e índices sintéticos, con el fin de facilitar su comparación entre distintos ámbitos territoriales, potenciar sinergias y optimizar el uso de recursos.

Palabras clave: sostenibilidad territorial; ordenación del territorio; sistemas de evaluación y seguimiento; indicadores.

\begin{abstract}
The gradual process of Europeanisation of spatial planning has promoted the inclusion of new sustainable development goals and evaluation and monitoring systems within regional plans. However, in Spain, unlike other countries with national guidelines, this inclusion has not been done systematically. This paper aims to verify to what extent and how these evaluation and monitoring systems are being integrated within Spanish regional plans paying special attention to the use of indicators. The review of these current plans allows us to state that Spain is still in its initial stages, since ten of its seventeen Autonomous Communities have developed specific evaluation and monitoring programs, including the elaboration of plans and reports, of which only five have actually developed and implemented the use of indicators. In addition, the approaches used differ significantly between each other, both in the conceptualization of what should be measured, i.e. how sustainability is defined, and in the number and type of the selected assessment indicators. In the future, a higher convergence in the definition of evaluation standards and guidelines that include the use of indicators and synthetic indexes is advised, in order to ease comparison between different planning areas, enhance synergies and optimize resources.
\end{abstract}

Keywords: territorial sustainability; spatial planning; evaluation and monitoring systems; indicators.

\title{
1 Marco teórico
}

A pesar de la clara apuesta por incluir el desarrollo territorial sostenible como objetivo clave de los principales documentos de ordenación europeos desde los años 90, el modelo de desarrollo urbano y territorial implantado en España a partir de la segunda mitad de los años 80 y hasta el comienzo de la crisis inmobiliaria y financiera en 2008 ha presentado claros signos de ineficiencia social, económica y ambiental. Este modelo, facilitado por regulaciones que propiciaron una 
expansión urbana sobre el territorio sin precedentes (Benach \& Walliser, 2011), se ha basado en gran medida en un crecimiento disperso, laxo y extensivo que, como se ha demostrado en otras investigaciones, llevadas a cabo fundamentalmente en los Estados Unidos (Ewing, 1997; Brueckner, 2000; Ewing \& Hamidi, 2015), implica un alto grado de incompatibilidad con la sostenibilidad.

Cómo este proceso no ha podido ser revertido, o al menos minimizado por la legislación y el planeamiento urbano y territorial, es todavía un asunto de debate. Algunos autores han afirmado que los sucesivos cambios en el sistema legal y la divergencia en el planeamiento emanado de las distintas Comunidades Autónomas (CC. AA.) han generado una gran incertidumbre en los agentes privados, así como problemas de coordinación y gestión por parte de la Administración (Calderón \& García, 2017). Además, las debilidades en el sistema de gobernanza, tales como la dispersión del poder municipal, la inexistencia de entes administrativos a la misma escala de los procesos territoriales y la baja participación de la sociedad civil en la planificación (Seixas \& Albet, 2016), han sido también factores importantes en la generación de un modelo urbano y territorial no coincidente con los objetivos teóricos de un desarrollo más sostenible, que no ha podido ser encauzado por el sistema de planificación existente en España. Como señala Burriel (2008), en muchos casos el planeamiento territorial o bien ha sido inexistente o bien ha sido inadecuado, mientras que muchos Ayuntamientos, dada su debilidad económica, han cedido a la presión de los agentes privados a la hora de planificar su desarrollo futuro.

La existencia de un modelo de desarrollo poco sostenible, en un marco claramente influenciado por la política de planificación espacial de la Unión Europea, ha llevado a plantear la necesidad de utilizar nuevos enfoques de planificación en los que destaca la incorporación de mecanismos de evaluación y seguimiento territorial desde una óptica de sostenibilidad. Sin embargo, la ordenación territorial llevada a cabo tanto en Europa, como especialmente en España, ha adolecido de vaguedad conceptual sobre qué es y en qué consiste el desarrollo sostenible (Tanguay et al., 2010) y no ha conseguido desarrollar sistemas de evaluación y seguimiento metodológicamente consensuados.

Los sistemas de evaluación y seguimiento se conciben como herramientas de medida y valoración del rendimiento efectivo de proyectos, planes o políticas (UNDP, 2002) con el objetivo de mejorar su implementación, resultados e impactos. Así, mientras que las herramientas de monitorización proporcionan una medición continua del desarrollo de un proyecto, plan o política, la evaluación debe realizar una valoración independiente de los resultados o impactos, ya sea ésta realizada ex-ante o, más comúnmente, ex-post.

A nivel conceptual pueden diferenciarse dos grandes tipos de aproximaciones a la evaluación, derivadas de dos formas distintas de entender lo que es un plan, ya sea éste territorial o de otro tipo: la evaluación basada en la conformidad con el plan y la evaluación basada en el rendimiento 
del plan (Faludi, 2000, 2006). La evaluación basada en la conformidad con el plan se orienta a determinar si sus objetivos están siendo alcanzados gracias a las medidas que se derivan de él. El plan actúa como una imagen, más bien cerrada, de cómo debería ser la realidad planificada en el futuro. En cambio, en la evaluación basada en el rendimiento, el plan se entiende más bien como una guía estratégica cuyo éxito se evalúa en función de su utilidad a la hora de influir y coordinar las decisiones de la gran multitud de agentes implicados en el ámbito de actuación del plan. En esta dicotomía el plan sería, en el primer caso, un proyecto de cómo debería ser la realidad una vez éste haya sido aplicado, mientras que en el segundo caso el plan actúa más bien como un marco estratégico abierto que ayuda a la toma de decisiones.

Desde la perspectiva de los tipos de evaluación, Laurian et al. (2010), basándose en los trabajos anteriores de Lunt et al. (2003), clasifican éstos en cuatro clases principales: las evaluaciones dirigidas a los objetivos o metas, las evaluaciones dirigidas o basadas en la teoría, las evaluaciones basadas en el uso del plan por parte de los agentes y las evaluaciones ateóricas basadas en los datos. Las evaluaciones dirigidas a los objetivos intentan vincular las metas del plan con sus resultados. Parten por lo tanto de la hipótesis de que en los planes existen unos objetivos explícitos cuyo cumplimiento debería alcanzarse gracias a las políticas o proyectos emanados del plan. Esta es seguramente la aproximación a la evaluación más común en los planes territoriales. En segundo lugar, las evaluaciones basadas en la teoría se centran en mayor medida en intentar establecer las relaciones que existen entre objetivos y resultados (Rogers et al., 2000). Estas relaciones son muchas veces difíciles de precisar, es decir, es complicado afirmar que una medida concreta y un resultado estén directamente relacionados sin considerar la existencia de otros factores no controlados. Por lo tanto, las evaluaciones basadas en la teoría intentan guiarse por un marco lógico que permita diferenciar los impactos directos de los indirectos y las consecuencias buscadas de las no buscadas con el objetivo de tener una imagen clara de los resultados que se derivan de forma efectiva del plan. Una tercera forma de evaluación se centra más bien en el uso del plan por parte de los agentes implicados en el mismo que en la relación entre objetivos y resultados. Este tipo de evaluación estaría más cercana a la conceptualización del plan basada en el rendimiento señalada por Faludi (2006). Esta aproximación se ha conocido también como de cuarta generación (Guba \& Lincoln, 1989) al centrarse más en los intereses y percepciones de los agentes sobre la evolución del plan que en la medición de la relación entre objetivos y resultados. Finalmente, la evaluación basada en los datos es propuesta por Laurian et al. (2010) como un tipo de evaluación focalizada en la cuantificación de los cambios territoriales, pero incapaz de responder de forma efectiva a la pregunta de si esos cambios se derivan de las acciones del plan. Sería por lo tanto el caso de una evaluación con un bajo o nulo contenido teórico donde la medición se hace más bien en función de los indicadores y datos que se encuentran disponibles y no a la inversa, como resultado de expectativas teóricas sobre los impactos que debería implicar el plan. 
En todas las aproximaciones la ausencia de metodologías estandarizadas y ampliamente aceptadas (Guyadeen \& Seasons, 2018, p. 105) ha llevado a varios autores a hacer propuestas en este sentido. Estas propuestas usualmente intentan combinar, de forma pragmática, distintos aspectos de los tipos de evaluación ya señalados. Entre éstas se encuentra la Plan-Outcome-Evaluation (POE) de Laurian et al. (2010), la cual combina elementos de las evaluaciones dirigidas a objetivos y basadas en la teoría. Esta metodología se basa en tres pasos principales: 1) la clarificación de los elementos del plan, es decir, de los objetivos, metas, políticas, regulaciones, etc. 2) la comparación de los objetivos del plan con los resultados observados mediante el uso de indicadores y 3) la utilización del conocimiento de expertos para identificar si los resultados observados han sido generados por las medidas derivadas del plan o por otros factores. Los autores aplicaron la metodología POE a la evaluación de varios planes locales de Nueva Zelanda. Oliveira \& Pinho (2010) propusieron también una metodología denominada Plan-Process-Results (PPR) construida alrededor de la evaluación de un plan en base al cumplimiento de nueve criterios organizados en tres dimensiones principales: la racionalidad ex-ante del plan (coherencia interna, coherencia externa, relevancia, interpretación), el rendimiento del plan (participación pública, utilización en la toma de decisiones) y la conformidad con el plan (compromiso con los recursos humanos y financieros, efectividad e incidencia del plan en el desarrollo urbano). Esta metodología fue aplicada a la evaluación de los planes directores municipales de Lisboa y Oporto.

En estas metodologías propuestas, y en otras similares (Laurian et al. 2004), un elemento común consiste en el uso de un sistema de indicadores que permita medir el grado de sostenibilidad de los territorios y de las actuaciones llevadas a cabo. En Europa la adopción de indicadores, como instrumentos dentro de los sistemas de evaluación y seguimiento, se ha dado sobre todo a partir de las decisiones adoptadas por los gobiernos nacionales en la conferencia de Naciones Unidas de Río de Janeiro de 1992. El Plan de acción de la Agenda 21 de Río contemplaba la necesidad de disponer de más información para la mejora de los procesos de toma de decisiones (UNCED, 1992). Las propias Naciones Unidas potenciaron el uso de indicadores de sostenibilidad con la publicación de una metodología para su desarrollo (UNCSD, 1996), posteriormente adoptada a nivel europeo por EuroStat (2001). En una línea similar, el European Spatial Planning Observatory Network (ESPON), establecido en el año 2000, fue fundado con el objetivo de recoger datos cuantitativos sobre la estructura territorial europea de cara a promover políticas con una mayor fundamentación (De Vries, 2009). Desde entonces ESPON ha ido elaborando un banco de datos y de indicadores especialmente desarrollado en el programa 2013-2020 con herramientas como la base de datos ESPON, el comparador entre áreas urbanas CityBench o el European Territorial Monitoring System. Sin embargo, el debate sobre la eficacia de estos indicadores para captar y medir realmente algo tan esquivo como es el concepto de sostenibilidad y la tensión entre la necesidad de contar con un conjunto de indicadores homogéneo que refleje a la vez las 
peculiaridades locales, aunque ha sido abordado por distintas iniciativas (Ambiente Italia, 2003), continúa abierto (Wong, 2006).

En el ámbito específico de los planes regionales, aunque existen diferencias entre los países europeos derivadas de sus particulares circunstancias culturales y políticas, en la mayoría de los casos se han ido desarrollando marcos orientativos de selección de indicadores definidos por organizaciones suprarregionales a nivel nacional, como es el caso de Inglaterra y Alemania. Estos marcos proporcionan directrices de carácter obligatorio, u orientativo para los organismos de planificación regional, usando indicadores que miden la implementación de las políticas de planificación y sus resultados o bien se organizan atendiendo al tema y la categoría de los objetivos del plan (Mascarenhas, 2012). Un caso representativo es el de Inglaterra, en donde se estableció un marco, basado en la formalización de una guía (ODPM, 2002) posteriormente revisada (DCLG, 2008), que contiene indicadores básicos para el seguimiento obligatorio de las estrategias de planificación aplicable a todas las regiones (Wong et al., 2008).

Estos sistemas presentan ciertas deficiencias de implementación en la práctica y dificultades de integración con la Evaluación Ambiental Estratégica (Mascarenhas, 2012). Los principales problemas que se atribuyen a los mecanismos de seguimiento de los planes regionales, y más concretamente a los sistemas de indicadores utilizados, están relacionados con el marco conceptual y metodológico, como son la falta de fuentes y bases de datos comparables, la gran cantidad de indicadores manejados, la ausencia de representatividad y categorización de los indicadores, la escasa vinculación entre los objetivos y los indicadores propuestos y la falta, en definitiva, de un procedimiento claro y sistemático de cómo debe abordarse el proceso de seguimiento del plan. No obstante, su desarrollo ha tenido una clara influencia en la formación de una arquitectura de indicadores que permita realizar el seguimiento de la implementación de los planes y del grado de consecución del desarrollo sostenible atendiendo a la demanda de protección ambiental, desarrollo económico y equidad social, lo que ha supuesto un avance en las prácticas de las políticas territoriales de los organismos de planificación regional.

En los apartados siguientes se analiza brevemente la evolución histórica de la planificación territorial en España y su proceso de europeización, resaltando los cambios conceptuales y metodológicos ligados a los distintos momentos históricos, y cómo éstos han cristalizado en las prácticas actuales, en las que la evaluación y seguimiento de los instrumentos de ordenación, mediante la inclusión de sistema de indicadores, todavía se encuentra en sus fases iniciales. Para ello se revisan, a continuación, los planes de ordenación de rango superior presentes en las diecisiete Comunidades Autónomas españolas y se examina si estos instrumentos cuentan con algún tipo de mecanismo de evaluación y/o de seguimiento que permita valorar sus resultados y la evolución de la sostenibilidad territorial. Además, se analiza si estos sistemas incluyen el uso de indicadores y se comentan las convergencias y divergencias en las aproximaciones utilizadas. Por último, se recogen las 
conclusiones del estudio y se realizan una serie de recomendaciones para la mejora de los sistemas de indicadores como instrumentos de evaluación y seguimiento y como herramientas clave para el apoyo a la toma de decisiones en el campo de la planificación territorial.

\section{La introducción de mecanismos de evaluación y seguimiento en España}

\subsection{La evolución de la ordenación del territorio española hasta la incorporación de sistemas de evaluación y seguimiento}

Un análisis de la larga trayectoria de la ordenación del territorio en España permite conocer cómo han evolucionado los planes, tanto desde el punto de vista teórico-conceptual como desde la perspectiva práctica del desarrollo metodológico de las figuras de planeamiento. De un lado, se ha pasado de la consideración de la planificación como un instrumento cuyo objetivo principal era el desarrollo económico a otro cuya preocupación fundamental es la sostenibilidad. Además, los planes han pasado de ser únicamente documentos propositivos a incluir el seguimiento de la eficacia y eficiencia de las medidas propuestas.

En España la institucionalización del planeamiento se produjo en 1956, año en el que se aprobó el primer marco jurídico urbanístico estatal, la Ley sobre el Régimen del Suelo y Ordenación Urbana (Ponce, 2004; Terán, 1982). Bajo esta ley prevaleció la planificación física o planeamiento urbanístico, imponiéndose un urbanismo localista, que no recogía las necesidades de articulación territorial, ni posibilitaba la integración económica y el reequilibrio territorial en ámbitos superiores al propiamente urbano o municipal.

La ordenación del territorio concebida como marco integrador de la planificación física en íntima conexión con la planificación económica, y como instrumento de reequilibrio poblacional, ambiental y económico (González \& Martínez, 1980) quedó marginada en la legislación urbanística de 1956, apareciendo como novedad en la Ley de Reforma de 1975 (y su Texto Refundido, el RD 1346/1976) y en el Reglamento de Planeamiento de 1978, representada en la figura de los Planes Directores Territoriales de Coordinación (PDTC), cuyo objetivo era establecer las directrices para la ordenación del territorio. Los intentos de llevarlos a la práctica, no obstante, supusieron un fracaso al que pudo contribuir, junto al establecimiento del Estado de las Autonomías, su planteamiento interno, muy concreto para aplicarlos a espacios de ámbito regional (Nogués, 1992).

Tras la configuración del Estado autonómico español, el desarrollo del marco jurídico general establecido por esta ley se superpone al traspaso de competencias a las Comunidades Autónomas, en virtud de la cual se redactan las leyes autonómicas (Feria et al., 2005), lo que ha facilitado una profunda transformación del Derecho Urbanístico español. Estas primeras leyes de ordenación del territorio autonómicas aprobadas entre 1983 y 2001 presentan una serie de elementos comunes, por cuanto se basan en la legislación estatal anterior, así como en las leyes que van aprobando 
otras CC. AA. En todos los casos, se adopta una visión jerárquica en la que se prevén, en la cúspide del sistema, las Estrategias, Directrices o Planes Regionales de Ordenación del Territorio, cuya función principal es definir el modelo territorial deseable (véase la Tabla 1 con los principales instrumentos de Ordenación Territorial de ámbito regional según año de aprobación y la Figura 1 con su localización).

Los planes de este periodo se fundamentan en la Carta Europea de Ordenación del Territorio (Consejo de Europa, 1983). Este documento define la ordenación del territorio como la expresión espacial de la política económica, social y ambiental de la sociedad, y establece como objetivos fundamentales el desarrollo socioeconómico equilibrado, la mejora de la calidad de vida, la gestión responsable de los recursos naturales y la utilización racional de territorio.

Se inicia así un proceso de europeización de la ordenación del territorio en España que tiene un punto de inflexión especialmente significativo a finales del siglo XX, con la aprobación de la Estrategia Territorial Europea (Comisión Europea, 1999). A pesar del escepticismo de España durante el proceso de preparación (Farinós et al., 2005) y de su carácter meramente indicativo, esta Estrategia, y en general la política europea de planificación espacial, ha tenido una gran incidencia en los cambios hacia un sistema de ordenación territorial basado en la planificación estratégica que supere la preponderancia del planeamiento urbanístico y la desconexión con la planificación económica (Giannakourou, 2005). Además, ha propiciado la incorporación de objetivos de desarrollo territorial sostenible en las nuevas estrategias y planes estratégicos autonómicos, siguiendo la estela de planes similares de la Unión Europea y del mundo occidental (Elorrieta et al., 2016). Este es el caso de las Estrategias Territoriales aprobadas en Aragón, Comunitat Valenciana y Navarra (véase Tabla 1 y Figura 1), así como de los planes más recientes o que se encuentran en fase de tramitación, como es el caso de Cantabria.

Tabla 1.Instrumentos de Ordenación Territorial de ámbito regional de las CC.AA. españolas

\begin{tabular}{|l|l|c|}
\hline \multicolumn{1}{|c|}{ C. A. } & \multicolumn{1}{|c|}{ INSTRUMENTO DE ORDENACIÓN TERRITORIAL } & $\begin{array}{c}\text { AÑO DE } \\
\text { APROBACIÓN }\end{array}$ \\
\hline Andalucía & Plan de Ordenación del Territorio de Andalucía (POTA) & 2006 \\
\hline Aragón & Estrategia de Ordenación Territorial de Aragón (EOTA) & 2014 \\
\hline Asturias & Directrices Regionales de Ordenación del Territorio (DROT) & $\begin{array}{c}1991 \text { (Revisión } \\
2006 \text { y 2016) }\end{array}$ \\
\hline Islas Baleares & $\begin{array}{l}\text { Directrices de Ordenación Territorial de las Illes Balears } \\
\text { (DOT) }\end{array}$ & 1999 \\
\hline Canarias & $\begin{array}{l}\text { Directrices de Ordenación General y Directrices de } \\
\text { Ordenación del Turismo de Canarias }\end{array}$ & $\begin{array}{c}2003 \\
\text { (Derogadas) }\end{array}$ \\
\hline Cantabria & Plan de Ordenación del Territorio de Cantabria (PROT) & En tramitación* \\
\hline Castilla y León & $\begin{array}{l}\text { Directrices Esenciales de Ordenación del Territorio de } \\
\text { Castilla y León }\end{array}$ & 2008 \\
\hline $\begin{array}{l}\text { Castilla-La } \\
\text { Mancha }\end{array}$ & $\begin{array}{l}\text { Plan de Ordenación del Territorio de Castilla-La Mancha } \\
\text { (POT) }\end{array}$ & En tramitación \\
\hline
\end{tabular}


Tabla 1. Continuación

\begin{tabular}{|l|l|c|}
\hline \multicolumn{1}{|c|}{ C. A. } & \multicolumn{1}{|c|}{ INSTRUMENTO DE ORDENACIÓN TERRITORIAL } & $\begin{array}{c}\text { AÑO DE } \\
\text { APROBACIÓN }\end{array}$ \\
\hline Cataluña & Plan Territorial General de Cataluña (PTGC) & 1995 \\
\hline $\begin{array}{l}\text { Comunitat } \\
\text { Valenciana }\end{array}$ & Estrategia Territorial Comunitat Valenciana (ETCV) & 2011 \\
\hline Extremadura & $\begin{array}{l}\text { Directrices de Ordenación Territorial de Extremadura } \\
\text { (DOTEX) }\end{array}$ & En elaboración \\
\hline Galicia & Directrices de Ordenación Territorial (DOT) & 2011 \\
\hline La Rioja & Estrategia Territorial de La Rioja & En tramitación \\
\hline $\begin{array}{l}\text { Comunidad de } \\
\text { Madrid }\end{array}$ & Plan Regional de Estrategia Territorial & $\begin{array}{c}\text { Aprobadas las } \\
\text { Bases en 1996 }\end{array}$ \\
\hline $\begin{array}{l}\text { Región de } \\
\text { Murcia }\end{array}$ & Directrices Regionales de Ordenación Territorial & $\begin{array}{c}\text { Prevista su } \\
\text { elaboración }\end{array}$ \\
\hline Navarra & Estrategia Territorial de Navarra (ETN) & 2005 \\
\hline País Vasco & $\begin{array}{l}\text { Directrices de Ordenación Territorial del País Vasco (CAPV- } \\
\text { DOT) }\end{array}$ & $\begin{array}{l}\text { 1997 (Revisión } \\
2018 \text { en } \\
\text { tramitación) }\end{array}$ \\
\hline
\end{tabular}

Leyenda: En el caso de Cantabria, la documentación del PROT (abril 2018) deberá ser sometida a aprobación inicial por la Comisión Regional de Ordenación de Territorio y Urbanismo de Cantabria.

Fuente: elaboración propia a partir de consultas en las webs de las consejerías autonómicas competentes en materia de ordenación territorial y el Ministerio de Medio Ambiente (2008)

Figura 1. Mapa de localización de los Instrumentos de Ordenación Territorial de las CC. AA. españolas

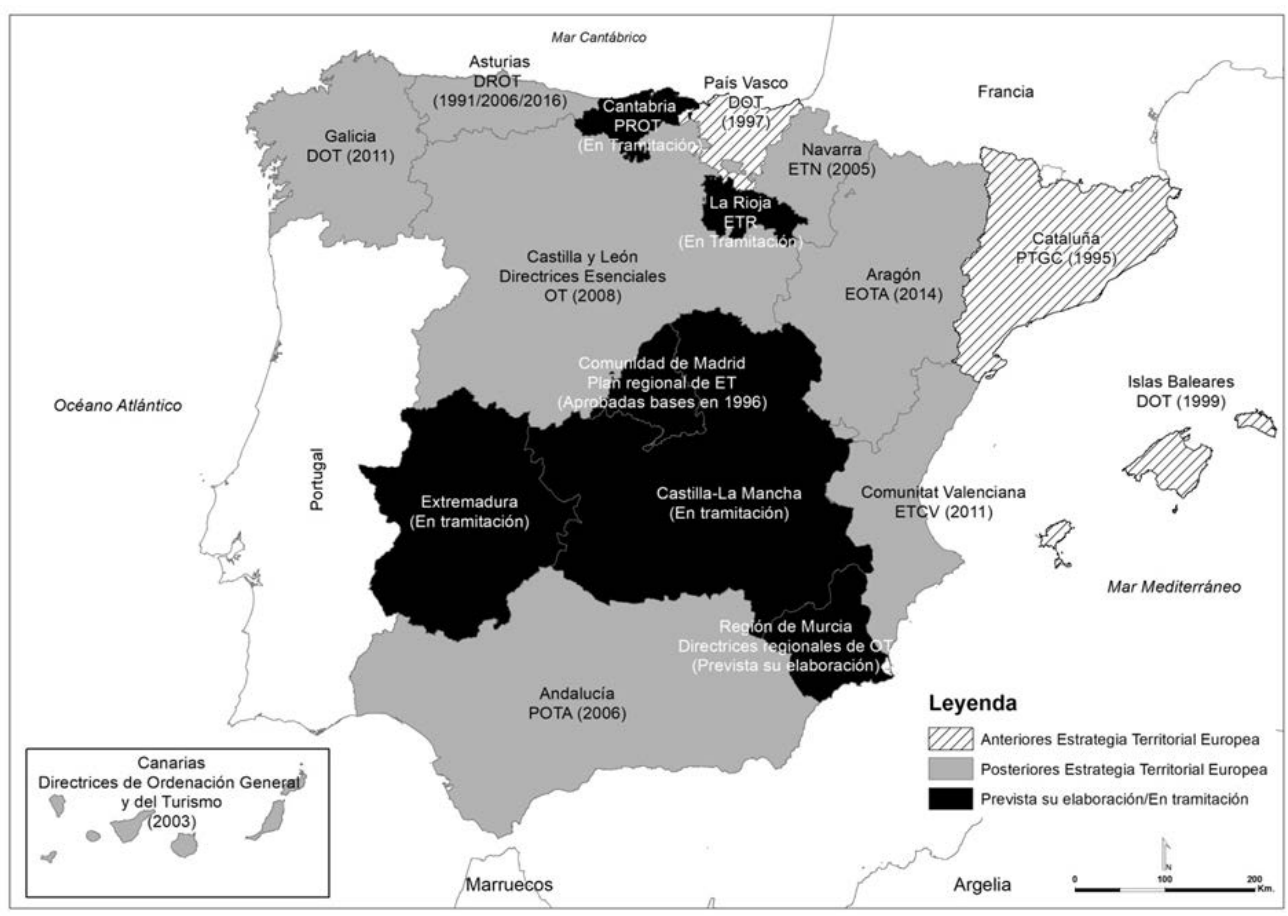

Fuente: elaboración propia a partir de consultas en las webs de las consejerías autonómicas competentes en materia de ordenación territorial y el Ministerio de Medio Ambiente (2008) 
Esta nueva forma de concebir la ordenación territorial conlleva la aparición de nuevos paradigmas de gobernanza territorial a nivel regional. En el nuevo planteamiento, la planificación se considera abierta, en el sentido mencionado por Faludi (2000, p. 316), puesto que el plan abre paso a un proceso que posibilita la evaluación y seguimiento continuo del progreso de implementación, el reestudio de los contenidos y la propuesta de nuevas políticas de cara al futuro. Esta nueva aproximación también viene acompañada de una creciente demanda de nuevos métodos y herramientas que permiten medir los resultados de las políticas de planificación espacial.

\subsection{La utilización de sistemas de evaluación y seguimiento mediante indicadores}

Aun considerando la diversidad de concepciones y las dificultades metodológicas de los sistemas de evaluación y seguimiento señaladas anteriormente, poco a poco las leyes y planes de las CC. AA. españolas, especialmente las más recientes, están incorporando este tipo de mecanismos de control (Elorrieta et al., 2016). Entre estos mecanismos se encuentran (Segura \& Pedregal, 2017; Farinós, 2008): las comisiones de seguimiento, los informes periódicos de seguimiento, la consulta y participación pública, la evaluación ambiental estratégica, la evaluación de impacto territorial, la creación de observatorios territoriales (Valenzuela \& Soria, 2011), los sistemas de información espacial y los sistemas de indicadores territoriales.

La introducción de estos sistemas de evaluación y seguimiento comienza a producirse en documentos de ordenación posteriores al cambio de siglo, bajo la influencia de la Estrategia Territorial Europea, de la idea de evaluación de impacto territorial (Farinós, 2011) y del concepto de una planificación más basada en la evidencia (Faludi \& Waterhout, 2006). Los sistemas evaluación y seguimiento pueden contemplarse además como un impulso a las mejoras, en términos de gobernanza, que deben realizarse para que la política de ordenación del territorio en España sea realmente efectiva. Estos sistemas pueden colaborar en la ejecución de los planes, fomentando la coordinación y cooperación entre los agentes públicos y privados para garantizar que las políticas emanadas del plan se materialicen de forma eficaz y eficiente (Hildenbrand, 2006, p. 112; Farinós, 2014, p. 337).

En el caso español, diez de las diecisiete CC. AA. contemplan la necesidad de realizar un seguimiento, elaborando planes o informes, e incluyendo en algunos casos entre sus herramientas de evaluación y seguimiento los sistemas de indicadores territoriales (Tabla 2). Los indicadores territoriales han sido considerados herramientas de gran importancia para la evaluación y seguimiento de los planes al permitir cuantificar la evolución territorial en términos de sostenibilidad, comparar ésta con la evolución de otros territorios y servir como datos de entrada para la elaboración de los informes periódicos de seguimiento. En lo que respecta al uso de dichos indicadores, tres comunidades los consideran de forma explícita en sus legislaciones autonómicas y en sus documentos de ordenación, Aragón, Navarra y Valencia; dos solamente en su legislación, 
La Rioja e Islas Baleares; mientras cinco recogen la necesidad de su aplicación como parte del seguimiento de su documento de ordenación regional, Andalucía, Asturias, Cantabria, Galicia y País Vasco (Tabla 2).

En el resto de Comunidades Autónomas españolas que contemplan el uso de indicadores, éstos responden a la medición de la calidad y sostenibilidad ambiental. Como señalan Segura \& Pedregal (2017), todas las leyes regionales incluyen el requisito de realizar una evaluación ambiental estratégica de los instrumentos de Ordenación del Territorio derivada de la Directiva Europea de Evaluación Ambiental Estratégica (European Parliament and of the Council, 2001). Esta evaluación debe contar con un programa de vigilancia ambiental que incluya medidas de evaluación y seguimiento mediante indicadores. Estos sistemas, sin embargo, todavía están siendo implementados en las CC.AA. españolas y en general no se han integrado en la evaluación y seguimiento de los instrumentos de Ordenación Territorial. Este es el caso por ejemplo de Castilla y León.

En el momento actual, únicamente cinco de las diez CC.AA. antes citadas, Aragón, Galicia, Navarra, País Vasco y Valencia han realizado labores de recopilación efectiva de información, y de medición de indicadores territoriales (Tabla 2). Sin embargo, su uso no ha sido homogéneo, pudiendo detectarse tanto similitudes como diferencias en los esquemas adoptados a la hora de conceptualizar la sostenibilidad y de definir los objetivos principales de la ordenación del territorio. En el siguiente apartado se exponen los indicadores utilizados en cada una de estas cinco CC. AA., como parte clave de sus sistemas de evaluación y seguimiento territorial.

Tabla 2. Evaluación y seguimiento en los Instrumentos de Ordenación Territorial de las CC. AA. españolas mediante informes e indicadores

\begin{tabular}{|l|l|l|}
\hline \multicolumn{1}{|c|}{ C. A. } & $\begin{array}{l}\text { INFORMES DE } \\
\text { SEGUIMIENTO } \\
\text { (AÑO) }\end{array}$ & DEFINICIÓN DEL PLAN DE SEGUIMIENTO E INDICADORES \\
\hline Andalucía & NO & $\begin{array}{l}\text { El POTA contempla la elaboración del Plan de Seguimiento y la } \\
\text { utilización de indicadores sobre la Estructura Territorial y la } \\
\text { Sostenibilidad, pero no los define. }\end{array}$ \\
\hline Aragón & NO & $\begin{array}{l}\text { La EOTA contempla la elaboración del Plan de Seguimiento y } \\
\text { define } 150 \text { indicadores calculados para la situación actual y } \\
\text { estableciendo su valor objetivo a futuro. Todavía no existe } \\
\text { informe de seguimiento. }\end{array}$ \\
\hline $\begin{array}{l}\text { Principado de } \\
\text { Asturias }\end{array}$ & NO & $\begin{array}{l}\text { El avance de la revisión de las DROT contempla su seguimiento } \\
\text { mediante la utilización de indicadores, pero no los define. }\end{array}$ \\
\hline Islas Baleares & NO & $\begin{array}{l}\text { Cuenta con un sistema de indicadores de sostenibilidad para el } \\
\text { seguimiento de las DOT aprobado, que define 25 indicadores. } \\
\text { No los calcula ni dispone de informes de seguimiento. }\end{array}$ \\
\hline Islas Canarias & NO & $\begin{array}{l}\text { El PROT contempla la elaboración del Plan de Seguimiento y la } \\
\text { utilización de indicadores, pero no los define. }\end{array}$ \\
\hline Cantabria & NO &
\end{tabular}


Tabla 2. Continuación

\begin{tabular}{|c|c|c|}
\hline C. A. & $\begin{array}{l}\text { INFORMES DE } \\
\text { SEGUIMIENTO } \\
\text { (AÑO) }\end{array}$ & DEFINICIÓN DEL PLAN DE SEGUIMIENTO E INDICADORES \\
\hline $\begin{array}{l}\text { Castilla y } \\
\text { León }\end{array}$ & NO & $\begin{array}{l}\text { La Comunidad cuenta con un conjunto de Indicadores para la } \\
\text { evaluación de la sostenibilidad y la calidad ambiental. }\end{array}$ \\
\hline $\begin{array}{l}\text { Castilla-La } \\
\text { Mancha }\end{array}$ & $\mathrm{NO}$ & \\
\hline Cataluña & $\mathrm{NO}$ & $\begin{array}{l}\text { La Comunidad cuenta con } 19 \text { Indicadores sobre Medio } \\
\text { Ambiente y Sostenibilidad. }\end{array}$ \\
\hline $\begin{array}{l}\text { Comunitat } \\
\text { Valenciana }\end{array}$ & $\mathrm{NO}$ & $\begin{array}{l}\text { La Estrategia Territorial contempla la elaboración del Plan de } \\
\text { Seguimiento y define } 100 \text { indicadores, que calcula en la } \\
\text { situación actual, estableciendo su valor objetivo a futuro. } \\
\text { Todavía no existe informe de seguimiento. }\end{array}$ \\
\hline Extremadura & $\mathrm{NO}$ & \\
\hline Galicia & SI (2013) & $\begin{array}{l}\text { Cuenta con Plan de Seguimiento aprobado y un conjunto de } 32 \\
\text { Indicadores de Sostenibilidad Territorial. Dispone de cuatro } \\
\text { informes de seguimiento. }\end{array}$ \\
\hline La Rioja & $\mathrm{NO}$ & $\begin{array}{l}\text { La Ley } 5 / 2006 \text { contempla la utilización de indicadores para el } \\
\text { seguimiento, pero no los define. }\end{array}$ \\
\hline $\begin{array}{l}\text { Comunidad } \\
\text { de Madrid }\end{array}$ & $\mathrm{NO}$ & $\begin{array}{l}\text { La Comunidad cuenta con Indicadores Ambientales, pero no } \\
\text { integrados en la Ordenación Territorial. }\end{array}$ \\
\hline $\begin{array}{l}\text { Región de } \\
\text { Murcia }\end{array}$ & $\mathrm{NO}$ & \\
\hline $\begin{array}{l}\text { Comunidad } \\
\text { Foral de } \\
\text { Navarra }\end{array}$ & $\begin{array}{l}\text { SI }(2003, \\
\text { modificado en } \\
2015)\end{array}$ & $\begin{array}{l}\text { La Ley Foral establece el seguimiento de la Estrategia Territorial } \\
\text { mediante indicadores del Observatorio Territorial de Navarra. } \\
\text { Cuenta con Memorias bienales desde } 2005 \text { e Informes de } \\
\text { seguimiento basados en } 29 \text { indicadores. }\end{array}$ \\
\hline País Vasco & SI (2016) & $\begin{array}{l}\text { La revisión de las DOT define el conjunto de } 6 \text { Indicadores de } \\
\text { Sostenibilidad Territorial y Urbanística. Dispone de dos informes } \\
\text { de resultados y un visor web. }\end{array}$ \\
\hline
\end{tabular}

Fuente: elaboración propia a partir de consultas a las webs de las consejerías autonómicas competentes en materia de ordenación territorial y los boletines oficiales autonómicos

\section{Los sistemas de indicadores de las Comunidades Autónomas de Navarra, Valencia, Galicia, Aragón y País Vasco}

\subsection{Evaluación de la Estrategia Territorial de Navarra (2003, modificado 2015)}

En la Comunidad Foral de Navarra, la Ley Foral 35/2002, de Ordenación del Territorio y Urbanismo, define la Estrategia Territorial de Navarra (ETN) como el instrumento básico de la ordenación territorial en la comunidad (Gobierno de Navarra, 2003). Esta estrategia es el primer documento de ordenación español -y de los primeros europeos- en aplicar los objetivos de competitividad, cohesión social y sostenibilidad, así como la metodología planteada en la Estrategia Territorial Europea (ETE) (Zarraluqui, 2003). Además, se incluye la necesidad de realizar una evaluación y seguimiento continuo del cumplimiento de los objetivos fijados por la ETN con la definición y el uso de indicadores territoriales y la creación del Observatorio Territorial de Navarra 
(OTN) encargado de su estimación y análisis. La periodicidad del seguimiento de la ETN fue definida por la Ley 35/2002 en dos años, estableciéndose posteriormente una frecuencia anual por la Ley Foral 5/2015, de medidas para favorecer el urbanismo sostenible, la renovación urbana y la actividad urbanística de Navarra. Esta ley incluye además la realización, cada cuatro años, de una memoria para el Gobierno y el Parlamento de Navarra sobre el grado de cumplimiento de las previsiones, la vigencia de las propuestas y la necesidad de revisión de la ETN (Gobierno de Navarra, 2003). Actualmente se dispone de cuatro memorias bienales, 2005-2006, 20072008, 2009-2010 y 2011-2012, y dos informes anuales, 2015 y 2016 (Nasuvinsa, 2017). Además, gracias al Decreto 1/2017 por el que se aprobó el Texto Refundido de la Ley de 2015, se crea el Consejo Social de Política Territorial (CSPT), un órgano de asesoramiento para la participación de diversos agentes en el seguimiento del desarrollo sostenible territorial de la Comunidad Autónoma (C. A.), el cual se apoya también en el OTN (Nasuvinsa, 2017).

La ETN definió un conjunto de 89 indicadores básicos, de situación y seguimiento, que agrupaba en siete indicadores sintéticos correspondientes a los criterios de competitividad output, competitividad input, cohesión social, sostenibilidad, policentrismo, accesibilidad a infraestructuras y conocimiento y gestión del patrimonio natural y cultural (Gobierno de Navarra, 2003; Zarraluqui, 2003; García, 2011), entendiendo el criterio de sostenibilidad solo desde su vertiente ambiental. A pesar de contar con la definición de estos indicadores las primeras memorias bienales, de 2005 2006 y 2007-2008, hicieron patente la necesidad de simplificar y hacer más comprensible la evaluación de los objetivos territoriales de la ETN, lo que derivó en el establecimiento, en 2011, del sistema de indicadores del OTN (2016) y la posterior modificación de la ley de ordenación.

El plan de seguimiento anual, iniciado en 2015, cuenta finalmente con un total de 29 indicadores obtenidos del Observatorio Territorial de Navarra, organizados en seis, en lugar de siete, principios clave (Tabla 3), relacionados con el desarrollo equilibrado y sostenible, en el que las dos competitividades previas se agrupan en una y el criterio llamado de sostenibilidad se sustituye por otro denominado conservación (OTN, 2016; Nasuvinsa, 2017), lo que elimina posibles malentendidos en la definición de los componentes de la sostenibilidad global. Posteriormente el plan de seguimiento anual evalúa la evolución de estos indicadores y compara su estado con los valores para la Unión Europea de los 15 (UE-15), España y los retos territoriales europeos planteados por ESPON. Estos resultados son finalmente utilizados para la realización de propuestas de mejora de las políticas sectoriales y territoriales.

Así, por ejemplo, en el caso de la cohesión social se usan cinco indicadores: la dependencia senil, el número de inmigrantes de origen extranjero, el riesgo de pobreza, la actividad femenina y el abandono escolar temprano, al igual que en el criterio de accesibilidad, donde se detallan cinco indicadores que tratan la accesibilidad de la población a servicios esenciales como centros médicos 
o escolares o el acceso on-line de servicios administrativos. De este listado, llama la atención que no se trate de manera explícita la cuestión de la ocupación de suelo, tan relevante para la sostenibilidad. No obstante, sí se considera implícitamente, a través de un indicador sobre el grado de alteración del paisaje, el porcentaje de cada tipo que ha experimentado un cambio de uso del suelo en un periodo determinado, siendo los más afectados aquellos que se han destinado a la construcción de nuevas urbanizaciones e infraestructuras dado su carácter de emplazamientos idóneos para estos usos.

La aplicación de indicadores por parte de la C. A. de Navarra destaca, por lo tanto, por su clara conceptualización de la sostenibilidad y por evaluar su evolución de forma continua y comparada con otros marcos territoriales, estableciendo además órganos específicos responsables de realizar estas tareas y de la promoción de la participación. Estos órganos han realizado además un esfuerzo continuo por simplificar el sistema de indicadores con el objetivo de agilizar y fomentar su uso. Sin embargo, el sistema actual no define índices sintéticos que den una clasificación y una medición única de la situación de sostenibilidad en las distintas subáreas establecidas por la ETN. Estos indicadores sintéticos fueron eliminados, tras la modificación realizada en el sistema de indicadores en 2011, al no existir un método estandarizado para elaborarlos a nivel internacional (García, 2011).

\subsection{Seguimiento de la Estrategia Territorial de la Comunitat Valenciana (2011)}

La Ley 5/2014, de Ordenación del Territorio, Urbanismo y Paisaje de la Comunitat Valenciana, que sustituye a la Ley 4/2004 de Ordenación del Territorio y Paisaje, introduce como principales novedades la integración y armonización de todos los niveles sujetos a planificación estableciendo además un nuevo instrumento de elaboración y evaluación del plan de ordenación (BOE, 2014). Tras aportar una definición clara del desarrollo territorial y urbano sostenible y apostar por crecimientos más ligados a la compacidad y regeneración que a la dispersión, la ley establece la Estrategia Territorial como documento de mayor rango en la ordenación autonómica e incluye, como contenidos de la misma, la definición de indicadores para evaluar el cumplimiento de los objetivos territoriales estratégicos. La Estrategia Territorial de la Comunitat Valenciana, aprobada en 2011, contempla la elaboración de un informe de seguimiento del desarrollo y eficacia de la misma en atención a los 25 objetivos propuestos, relacionados con el sistema de asentamientos y las grandes ciudades, el mundo rural, los recursos naturales, económicos y culturales, la movilidad y conectividad, el cambio climático y la gestión y la gobernanza territorial (Tabla 3). El cumplimiento de estos objetivos es evaluado por un total de 100 indicadores, 4 por cada objetivo. Así, por ejemplo, la ocupación de suelo es tratada en el objetivo 23, en el que se precisa una ocupación racional y sostenible, y su cumplimiento evaluado a partir de indicadores concernientes al 
crecimiento de suelo residencial, la relevancia del tejido urbano continuo respecto del total o el porcentaje de zonas verdes en áreas urbanas (Generalitat Valenciana, 2015).

Por otro lado, en el caso de la movilidad, existe un objetivo concreto (19) en el que se especifican indicadores relativos a la oferta de transporte público y la disponibilidad de planes de movilidad. Otros indicadores relacionados con la movilidad son los definidos en el objetivo de conectividad (18), que se centra en los servicios ferroviarios y la accidentalidad, y en el de actividad económica (24), referente a la movilidad al trabajo.

Llama la atención en algunos casos la escasa relación entre los indicadores propuestos y el objetivo a perseguir. Este es el caso del objetivo de mejora de las condiciones de vida en el sistema rural (5), que mientras concreta la accesibilidad y la cohesión social en base a dotaciones como parte de las dimensiones de la ruralidad, sus cuatro metas e indicadores están ligados al crecimiento demográfico (Generalitat Valenciana, 2015, objetivo 5, p. 34).

El seguimiento de la Estrategia valenciana responde por lo tanto a una definición clara de la sostenibilidad y sus componentes y a la necesidad de una medición continua de su evolución. Además, los indicadores permiten fijar objetivos cuantitativos a la Estrategia en el horizonte 2030, si bien puede ser difícil atribuir el cumplimiento o incumplimiento de esos objetivos a las medidas derivadas de la propia Estrategia. La comparativa de la situación actual de partida de la C.A. se realiza en contraposición con los valores de otros países de la Unión Europea, y no con ámbitos territoriales más similares, y además en base a unos indicadores diferentes de los planteados para definir y evaluar las metas propuestas para cada objetivo. Al igual que en el caso de Navarra, no se plantean índices sintéticos que puedan clasificar la situación de los ámbitos internos de la C. A.

\subsection{Plan de Seguimiento de las Directrices de Ordenación del Territorio de Galicia (2013)}

El desarrollo de la legislación y planificación territorial gallega ha sido pobre, tal como señalan Lois \& Aldrey (2011), debido en buena parte a una falta de interés de la mayoría de su población tradicionalmente ligada a la cultura de la autoconstrucción. En contraposición al prolífico desarrollo legislativo urbanístico (10 leyes), en el caso de la ordenación territorial solamente una ley ha sido implementada en más de 30 años de autonomía, la Ley 10/1995, de Ordenación del Territorio de Galicia, una de las más tardías en España. Esta ley, redactada al amparo de los principios básicos de la Carta Europea de Ordenación del Territorio de 1983, ha tenido además que enfrentarse a un modelo y estrategia de desarrollo territorial completamente diferente, como es el propuesto por la Ley de Comarcalización de 1996, lo que ha ocasionado una serie de agravios traducidos en una escasa y demorada implementación. La Ley 10/1995 estableció como instrumento fundamental para su implementación las Directrices de Ordenación del Territorio (DOT); sin embargo, estas DOT, que presentaron sendos avances en 2004 y 2008, no fueron aprobadas hasta el año 2011 (Decreto 19/2011, BOE, 2011). A falta de documentos previos, y dada la tardanza en su 
elaboración, las DOT proponen una ordenación integral y equilibrada del territorio, incluyendo nuevos conceptos asociados al desarrollo sostenible, como el respeto por la calidad ambiental, el uso racional de los recursos y el aprovechamiento de las potencialidades de cada zona del territorio (Xunta de Galicia, 2011).

Posteriormente a la aprobación de las directrices, de acuerdo al Decreto 156/2012 de 12 de julio, se crea la Comisión de Seguimiento de las DOT para la elaboración del Plan de seguimiento de la sostenibilidad territorial de las mismas, en el marco del procedimiento de la Evaluación Ambiental Estratégica que establece la ley de ordenación del año 1995 y en respuesta a la determinación 10.1.19 de las DOT. El plan de seguimiento presenta una metodología para obtener periódicamente información sobre el estado de la estructura territorial de Galicia, de tal forma que sea posible evaluar el cumplimiento de los objetivos formulados por las DOT (Xunta de Galicia, 2013). Dando respuesta al plan de seguimiento, actualmente existen cuatro informes de los años 2013, 2015, 2016 y 2017 (Xunta de Galicia, 2018). Esta información se recoge a través de indicadores cuantitativos que tratan de dar respuesta a tres preguntas planteadas: ¿Qué grado de cumplimiento presentan las determinaciones emanadas de las DOT?, ¿las medidas son eficaces para incrementar la sostenibilidad? y ¿cómo percibe la ciudadanía estos desarrollos? Para ello, el plan de seguimiento define dos tipos de indicadores: los Indicadores de Desarrollo e Implantación (IDI), que buscan responder a la pregunta del grado de cumplimiento de las DOT, y los Indicadores de Sostenibilidad Territorial (IST), que buscan responder a la cuestión de la eficacia de las directrices para el incremento de la sostenibilidad.

Dentro de los IST, el plan de seguimiento diferencia siete grandes bloques de variables que conceptualizan la sostenibilidad territorial. Estos bloques, recogidos en la Tabla 3, se corresponden con los objetivos de consumo eficiente del suelo, integración paisajística, preservación de la funcionalidad de los ecosistemas, reconocimiento y valorización del patrimonio humano, aumento de la cohesión social/diversidad de funciones, máxima autosuficiencia de los flujos metabólicos y fomento de la movilidad sostenible, emanados de las DOT.

Para cada bloque o componente se establecen sub-bloques de variables consideradas aspectos clave de las directrices y, finalmente, indicadores de medición. Por ejemplo, el bloque sobre ocupación del territorio se divide en los sub-bloques: usos del suelo, distribución, intensidad y dispersión. A su vez estos cuatro sub-bloques presentan los indicadores: variación de la superficie de los usos del suelo, proximidad a núcleos de carácter funcional, saturación del suelo disponible, crecimiento previsto, grado de conurbación y viviendas familiares (Xunta de Galicia, 2013). Del mismo modo, existe un bloque de indicadores de movilidad para el que se plantea un único indicador, el reparto modal en la movilidad cotidiana, aunque la proximidad a núcleos de carácter funcional, considerada en el bloque anterior, también está relacionada con la accesibilidad. 
Adicionalmente cada uno de los objetivos del modelo territorial de las DOT se cruza con uno o varios indicadores para evaluar la evolución de su sostenibilidad territorial. Algunos sub-bloques pueden presentar además más de un indicador de medición. Para cada indicador, el plan de seguimiento detalla tanto su metodología de cálculo como las fuentes utilizadas.

El uso de indicadores de evaluación en el marco de las DOT gallegas está, por lo tanto, claramente adaptado a un marco conceptual explícito sobre la definición de la sostenibilidad territorial. La diferenciación entre indicadores IDI e IST permite separar de forma nítida la evaluación del cumplimiento de las determinaciones de las DOT de la evolución en la consecución de la sostenibilidad. Sin embargo, el plan de seguimiento tampoco cuenta con comparativas con otros ámbitos regionales o índices sintéticos que permitan evaluar de forma sencilla la evolución de la sostenibilidad en la región.

\subsection{Seguimiento de la Estrategia de Ordenación Territorial de Aragón (2014)}

La Comunidad Autónoma de Aragón aprobó la vigente Estrategia de Ordenación Territorial (EOTA) en el año 2014, como respuesta al requerimiento de la Ley 4/2009, de Ordenación del Territorio de Aragón (Gobierno de Aragón, 2014a). Esta Ley, que sustituye a la de 1992, incorporó como una de sus principales novedades la relevancia del desarrollo sostenible como objetivo de la ordenación y la sustitución de las Directrices Generales de 1998 por la Estrategia de Ordenación Territorial (Bielza, 2010). La ley, basada en la Estrategia Europea 2020, incluye también como mandato la definición de un sistema de seguimiento del cumplimiento de los objetivos de la EOTA que contemple la elaboración de un informe de seguimiento propuesto en un plazo de cuatro años, mediante la utilización de indicadores cuantitativos (Gobierno de Aragón, 2014a).

La EOTA pretende cumplir veinte objetivos generales (Tabla 3), que comprenden los relacionados con la ocupación del suelo, medio ambiente y paisaje, la economía y los aspectos sociodemográficos. Para cada uno de estos objetivos generales fija desde uno hasta trece indicadores específicos, de un total de 150, para monitorizar su evolución y determinar si la estructura territorial se está acercando o alejando del modelo territorial planteado. El Sistema de Indicadores Territoriales de Aragón (SITA) estima 132 de los 150 indicadores definidos por la EOTA y elabora informes anuales (IDEAragon, 2017). Los datos son recogidos, en su mayoría, por el Instituto Aragonés de Estadística y publicados gracias a la Infraestructura de Datos Espaciales de Aragón, cuyo uso permite la comparación y cooperación entre administraciones públicas y la participación pública, aspectos muy relevantes para la ordenación (Sauco et al., 2015).

Por ejemplo, el primer objetivo de la EOTA sobre promoción de la actividad económica propone medir la evolución de ésta a través de dos indicadores sobre el número de trabajadores empleados en actividades económicas consideradas estratégicas y no estratégicas en el medio rural (Gobierno de Aragón, 2014b, p. 1). Por otro lado, dos de los veinte objetivos de la EOTA tienen una clara 
relación con la movilidad/accesibilidad, comprendiendo 38 indicadores. El tercer objetivo sobre organización de los equipamientos analiza, mediante 17 indicadores, la accesibilidad de la población a distintos equipamientos básicos como centros de salud, institutos o de tres equipamientos básicos a la vez. Adicionalmente, el cuarto objetivo sobre facilitación de la movilidad contempla, mediante 31 indicadores, factores diversos de la movilidad, como la existencia en los distintos asentamientos de servicios de transporte público, la extensión de la red de transporte público o el número de municipios que presentan un plan de movilidad.

El sistema de indicadores de Aragón, al igual que el navarro, no evalúa específicamente, sin embargo, uno de los componentes esenciales de la sostenibilidad, la ocupación de suelo. No obstante, lo aborda de forma implícita al evaluar los objetivos del sistema de asentamientos, alojamiento y espacios homogéneos y funcionales, mediante indicadores tales como: el porcentaje de viviendas unifamiliares respecto a las viviendas en bloque, el stock de vivienda nueva respecto al parque de viviendas o el porcentaje de suelo delimitado y regulado respecto al suelo total de la región (Gobierno de Aragón, 2014b, p. 1).

El uso de indicadores en la ordenación territorial en el caso de Aragón es por lo tanto ambicioso, dada la diversidad de objetivos planteados y la cuantificación de todos ellos mediante 150 indicadores, la cifra más elevada de las presentes en las regiones españolas. Por el momento, los indicadores calculados no se comparan de forma sistemática con los presentes en otras regiones ni se establecen recomendaciones para su cumplimiento, si bien sí se justifican los valores objetivo a futuro en relación a otras regiones y planes sectoriales. El sistema actual cuenta además con un índice sintético de desarrollo territorial, el cual agrupa cinco índices parciales referentes a: actividad económica, alojamiento, equipamientos/servicios, movilidad y escenario vital/patrimonio territorial, y un índice sintético de sostenibilidad del desarrollo territorial, a partir de índices parciales de compatibilidad ambiental del desarrollo territorial, viabilidad económica y grado de cohesión social (Gobierno de Aragón, 2014b). Los índices parciales se agregan y ponderan posteriormente en función de un consenso entre distintos agentes territoriales.

\subsection{Sistema de Indicadores de Sostenibilidad Territorial y Urbanística del País Vasco (2016)}

La Ley 4/1990, de Ordenación del Territorio del País Vasco, primera ley como tal de la C. A., determinó como la figura clave de planificación territorial las Directrices de Ordenación Territorial (DOT), que fueron aprobadas por el Decreto 28/1997 (Lozano et al., 2015). El gran desarrollo de instrumentos de planificación al amparo de esta ley ha posicionado al País Vasco como comunidad ejemplar en la ordenación regional en España. Sin embargo, esta ordenación se ha caracterizado por una difícil relación institucional entre los diversos niveles administrativos (Juaristi, 2009; Lozano, 2004), vinculada a la existencia de competencias repartidas en materia gestión territorial entre la Comunidad y las diputaciones forales, discordancias con la competencia municipal de la 
planificación urbanística e incompatibilidades en la definición de ámbitos de actuación entre áreas funcionales y límites administrativos. Todas estas discrepancias han complicado la manera de abordar actuaciones sectoriales o territoriales parciales y han generado cambios en la jerarquía de los documentos, lo que ha derivado en retrasos en la aprobación y puesta en práctica de los distintos documentos de ordenación y repercutido indirectamente, en no pocas ocasiones, en la excesiva artificialización de la C.A. (Lozano et al., 2015).

Como resultado de este proceso, la revisión de las DOT, en espera de aprobación definitiva (Gobierno Vasco, 2016), son resultado claro de la preocupación de la Comunidad Autónoma del País Vasco, una región de fuerte carácter urbano con una densidad de población elevada (300 habitantes por kilómetro cuadrado), por controlar y coordinar el desarrollo del suelo por parte de los municipios. Esto ha llevado a que estas nuevas DOT centren como uno de sus objetivos principales de planificación, y por tanto de evaluación y seguimiento, la limitación de la transformación física del territorio y su artificialización, en lugar de medir el avance hacia la sostenibilidad territorial en un sentido amplio.

El sistema de indicadores territoriales propuesto por el Gobierno Vasco para el seguimiento de la evolución territorial y urbanística de la comunidad es, por consiguiente, mucho más reducido que en otras CC. AA., y se agrupa en tres componentes principales (Tabla 3): indicadores de contexto urbano y territorial, indicadores del impacto del desarrollo actual/previsto e indicadores de artificialización-calificación (Gobierno Vasco, 2017a). Estos tres grupos presentan un total de seis indicadores desagregados a distintos niveles territoriales: calificación del suelo, densidad de población, proporción de suelo urbanizable por tipo de suelo, incremento de viviendas previsto, proporción de viviendas por tipo de suelo y evolución del suelo según su calificación/artificialización entre los años 2005 y 2016.

La elaboración de los indicadores se realiza a partir de la información contenida en el sistema de información geográfica y banco de datos territoriales UDALPLAN, el cual recopila el planeamiento urbanístico de los municipios y los cambios que se van produciendo en el mismo (Gobierno Vasco, 2017a). Los indicadores se actualizan anualmente y tienen una resolución espacial variable llegando hasta el nivel municipal en algunos casos. Actualmente se encuentran disponibles dos informes (tablas estadísticas) de los resultados de esta evaluación, para los años 2016 y 2017 (Gobierno Vasco, 2017b). Es de señalar que los indicadores no se comparan sistemáticamente con la evolución en otros ámbitos territoriales (otras CC. AA. u otras regiones europeas). 
Tabla 3. Componentes/objetivos considerados en el seguimiento de los Instrumentos de Ordenación

\begin{tabular}{|c|c|c|c|c|}
\hline NAVARRA & VALENCIA & GALICIA & ARAGÓN & PAÍS VASCO \\
\hline Cohesión Social & Cohesión social & \multirow{5}{*}{$\begin{array}{l}\text { Sociedad y } \\
\text { Economía }\end{array}$} & Cohesión social & \\
\hline \multirow{4}{*}{ Competitividad } & Innovación & & Recursos Humanos & \\
\hline & $\begin{array}{l}\text { Actividad } \\
\text { económica }\end{array}$ & & $\begin{array}{l}\text { Actividad } \\
\text { Económica }\end{array}$ & \\
\hline & Turismo & & $\begin{array}{l}\text { Viabilidad } \\
\text { Económica }\end{array}$ & \\
\hline & Logística & & & \\
\hline \multirow[b]{2}{*}{ Conservación } & Paisaje & Paisaje & \multirow[b]{2}{*}{ Medio Ambiente } & \multirow{2}{*}{$\begin{array}{l}\text { Artificialización/ } \\
\text { calificación }\end{array}$} \\
\hline & $\begin{array}{l}\text { Patrimonio } \\
\text { ambiental }\end{array}$ & \multirow[b]{2}{*}{$\begin{array}{l}\text { Ocupación del } \\
\text { Territorio }\end{array}$} & & \\
\hline \multirow{4}{*}{$\begin{array}{l}\text { Patrimonio Natural y } \\
\text { Cultural }\end{array}$} & $\begin{array}{l}\text { Ocupación de } \\
\text { suelo }\end{array}$ & & $\begin{array}{l}\text { Escenario vital y } \\
\text { patrimonio territorial }\end{array}$ & \\
\hline & Litoral & $\begin{array}{l}\text { Patrimonio } \\
\text { Natural }\end{array}$ & \multirow{2}{*}{ Recursos Naturales } & \\
\hline & Patrimonio cultural & $\begin{array}{l}\text { Patrimonio } \\
\text { Cultural }\end{array}$ & & \\
\hline & Cambio climático & & & \\
\hline Accesibilidad & Movilidad & Movilidad & Movilidad & \\
\hline \multirow{6}{*}{ Policentrismo } & $\begin{array}{l}\text { Sistema de } \\
\text { ciudades }\end{array}$ & \multirow{6}{*}{ Metabolismo } & $\begin{array}{l}\text { Sistema de } \\
\text { Asentamientos }\end{array}$ & \multirow{4}{*}{$\begin{array}{l}\text { Contexto urbano y } \\
\text { territorial }\end{array}$} \\
\hline & Valencia & & $\begin{array}{l}\text { Equilibrio } \\
\text { demográfico }\end{array}$ & \\
\hline & Alicante y Elx & & Equilibrio Territorial & \\
\hline & Castellón & & $\begin{array}{l}\text { Espacios } \\
\text { homogéneos y } \\
\text { funcionales }\end{array}$ & \\
\hline & Sistema rural & & & \\
\hline & Vivienda & & Alojamiento & $\begin{array}{l}\text { Impacto del } \\
\text { desarrollo actual y } \\
\text { previsto }\end{array}$ \\
\hline & Infraestructuras & & Infraestructuras & \\
\hline & Conectividad & & & \\
\hline & & & Equipamientos & \\
\hline & Recursos hídricos & & Recursos Hídricos & \\
\hline & Energía & & $\begin{array}{l}\text { Recursos } \\
\text { Energéticos }\end{array}$ & \\
\hline & $\begin{array}{l}\text { Riesgos naturales e } \\
\text { inducidos }\end{array}$ & & $\begin{array}{l}\text { Riesgos Naturales e } \\
\text { Inducidos }\end{array}$ & \\
\hline & Gestión territorial & & & \\
\hline & $\begin{array}{l}\text { Gobernanza } \\
\text { territorial }\end{array}$ & & $\begin{array}{l}\text { Gobernanza } \\
\text { territorial }\end{array}$ & \\
\hline & & & $\begin{array}{l}\text { Régimen jurídico } \\
\text { del territorio }\end{array}$ & \\
\hline
\end{tabular}

Fuente: elaboración propia a partir de Generalitat Valenciana (2015); Gobierno de Aragón (2014b); Gobierno Vasco (2017a); Nasuvinsa (2017) y Xunta de Galicia (2013) 
3.5 Comparativa de los sistemas de indicadores de las Comunidades Autónomas de Navarra, Valencia, Galicia, Aragón y País Vasco.

La recopilación y el análisis de los sistemas de indicadores utilizados por los diversos documentos de planificación regional de las CC. AA. muestra que el enfoque de la evaluación y seguimiento se corresponde en gran medida con la primera de las aproximaciones definidas por Laurian et al. (2010), es decir, una evaluación dirigida a examinar el cumplimiento de objetivos o metas. Esta aproximación es especialmente clara en el caso de la C. A. de Galicia, en la que el propio sistema de indicadores se relaciona directamente con cada uno de los objetivos definidos en el plan (Xunta de Galicia, 2013), con el fin de valorar su grado de cumplimiento.

Por otro lado, este análisis demuestra la elevadísima desproporción existente en la configuración y definición de los sistemas de indicadores de las distintas CC. AA. Los cinco planes analizados contemplan entre 3 y 25 componentes o criterios de evaluación, que se traducen en una horquilla con entre 6 y 150 indicadores (Tabla 4).

Tabla 4. Características de los sistemas de indicadores de los Instrumentos de Ordenación

\begin{tabular}{|c|c|c|c|c|c|}
\hline & NAVARRA & VALENCIA & GALICIA & ARAGÓN & PAÍS VASCO \\
\hline $\begin{array}{l}\text { Escala temporal del } \\
\text { análisis }\end{array}$ & Anual & Anual & Anual & $\begin{array}{l}\text { Variable } \\
\text { (Trimestral, } \\
\text { Anual) }\end{array}$ & Anual \\
\hline $\begin{array}{l}\text { Escala espacial del } \\
\text { análisis }\end{array}$ & $\begin{array}{l}\text { Sub-áreas de } \\
\text { la ETN de } \\
\text { Navarra }\end{array}$ & Regional & $\begin{array}{l}\text { Variable } \\
\text { (Regional, } \\
\text { Provincial, } \\
\text { Distritos, } \\
\text { Municipal) }\end{array}$ & Regional & $\begin{array}{l}\text { Variable } \\
\text { (Provincias, } \\
\text { Áreas } \\
\text { Funcionales, } \\
\text { Comarcas, } \\
\text { Municipios) }\end{array}$ \\
\hline $\begin{array}{l}\text { Número de } \\
\text { componentes } \\
\text { considerados }\end{array}$ & 6 & 25 & 7 & 20 & 3 \\
\hline $\begin{array}{l}\text { Número de } \\
\text { indicadores }\end{array}$ & 29 & 100 & 32 & 150 & 6 \\
\hline $\begin{array}{l}\text { Uso de índices } \\
\text { sintéticos }\end{array}$ & No & No & No & Sí & No \\
\hline $\begin{array}{l}\text { Comparación con } \\
\text { otros ámbitos } \\
\text { territoriales }\end{array}$ & Sí & Sí & No & No & No \\
\hline
\end{tabular}

Fuente: elaboración propia

Existen también grandes diferencias en cuanto al tipo de indicadores utilizados, que van desde los de estricto carácter ambiental-territorial hasta los económicos y sociales, lo que significa que bajo el 
concepto de "sostenibilidad territorial", como ya ha destacado Elorreita et al. (2016), se encuadran dimensiones muy diferentes. Un claro ejemplo de estas convergencias y divergencias encontradas entre las distintas regiones es el tratamiento de la ocupación de suelo, que solo se considera de manera expresa en Galicia, Valencia y País Vasco, a pesar de ser un elemento esencial en la evolución de la estructura territorial.

Otro ejemplo es el de la movilidad/accesibilidad, la cual tiene un impacto tanto en la eficiencia como en la equidad y sostenibilidad territorial. Así, mientras en el País Vasco, al estar los indicadores centrados en la transformación física del suelo, no existe ninguna medida que pueda relacionarse directamente con la movilidad/accesibilidad y su incidencia en la estructura territorial, el sistema navarro diferencia un componente de accesibilidad relacionado con la accesibilidad de la población a los servicios básicos, el gallego y el valenciano distinguen un bloque u objetivo de movilidad, relativo al reparto modal y existencia de planes de movilidad, y Aragón dos, añadiendo al anterior de la accesibilidad de la población la evaluación de la tipología y calidad de los servicios e infraestructuras de transporte.

Otro aspecto relevante sobre los contrastes en la evaluación de la sostenibilidad es la realización de comparativas con otras regiones, sólo llevada a cabo en los casos de Navarra, Aragón y Valencia, aunque este último solo lo realiza en base a la situación de partida y frente a otros países en lugar de regiones, o la utilización de índices sintéticos, solo considerada en Aragón.

Puede decirse, por lo tanto, que entre los cinco sistemas de indicadores actualmente desarrollados en las CC. AA. españolas hay cierta diversidad de aproximaciones al problema de realizar el seguimiento de la implementación de los planes y evaluar su impacto en la sostenibilidad territorial. Sin embargo, a excepción del País Vasco, parece que existe una cierta convergencia entre dos tipos de aproximaciones en cuanto al número de objetivos e indicadores utilizados, por un lado, los casos de Navarra y Galicia y, por otro, los de Valencia y Aragón.

\section{Conclusiones}

En esta investigación se ha realizado una breve síntesis de la conceptualización actual de los sistemas de evaluación y seguimiento aplicados a proyectos, planes o políticas. La revisión de este marco teórico ha permitido constatar que existen diversas concepciones de lo que es un sistema de evaluación y seguimiento, sin que exista actualmente una metodología consensuada a nivel internacional, si bien todas ellas suelen implicar el uso de indicadores territoriales como elemento clave.

En el caso español, en donde hasta la fecha únicamente dos terceras partes de las CC. AA. han logrado aprobar y poner en funcionamiento su instrumento de ordenación territorial regional, la evolución de los planes de ordenación del territorio, desde la institucionalización del planeamiento 
hasta el momento actual, ha estado marcada por la progresiva influencia europea. En el marco de esta evolución, patente en el contenido y documentación de los planes, se ha hecho especial hincapié en la incorporación de mecanismos de control, analizando las primeras aproximaciones que se están llevando a cabo en las Comunidades Autónomas españolas en el ámbito de la evaluación y seguimiento de la sostenibilidad territorial mediante el uso de indicadores. La importancia dada a este proceso de evaluación y seguimiento constituye un elemento novedoso de los últimos años, que supone un intento de superar una práctica que ha sido habitual de la ordenación del territorio en España hasta hace muy poco, como es la falta de una planificación que no sólo se limite a proponer un modelo territorial, sino que también evalúe y monitorice periódicamente su grado de cumplimiento.

Las actividades de evaluación y seguimiento se entienden como un mecanismo esencial del proceso de planificación, que proporciona información importante para la toma de decisiones, ya que permite determinar si los fines del plan están siendo alcanzados y, en su caso, establecer las medidas necesarias para ello. Sin embargo, las actuaciones de evaluación y seguimiento llevadas a cabo hasta ahora en los planes regionales españoles han sido limitadas, debido tanto a la falta de recursos y voluntad política (Segura \& Pedregal, 2017, p. 11) como a la ausencia o deficiencia de un marco conceptual y metodológico adecuado.

En España, no existe un marco comúnmente aceptado para evaluar y realizar el seguimiento de forma efectiva de los resultados de la planificación. De la investigación realizada se puede concluir que los sistemas que se están desarrollado tienden a encuadrase en un tipo basado en la conformidad con el plan y orientado a vincular sus objetivos y medidas con los resultados observados de la evolución territorial. Sin embargo, la falta de contenido conceptual de estos sistemas dificulta la implementación de metodologías basadas en datos que atribuyan, con certitud técnica, los cambios territoriales observados a las acciones derivadas del plan.

Lo que sí se acepta de forma bastante generalizada es que los sistemas de indicadores deben desempeñar un papel importante en la evaluación y seguimiento de los planes, aunque la selección de indicadores apropiados y valores de referencia siga siendo un desafío. El uso de indicadores representativos facilita el adecuado seguimiento de la evolución territorial de las CC. AA. y la valoración cuantitativa del cumplimiento de las previsiones de los planes, sirviendo de base para su actualización, modificación o revisión. No obstante, la pluralidad y diversidad de indicadores propuestos o establecidos pone de manifiesto la escasa univocidad de criterios entre las CC. AA. Los indicadores han sido tan diversos que del análisis conjunto difícilmente puede extraerse una opción clara, ni del número de indicadores ni de la estructura u objetivos a considerar, de cara a obtener datos de valor que posibiliten una reflexión fundamentada orientada a la planificación. 
Esta investigación plantea la necesidad de desarrollar un marco de evaluación y seguimiento coherente destinado a monitorizar los resultados de los planes regionales, que incluya un procedimiento claro, sistemático y teóricamente fundamentado, junto a un conjunto de indicadores representativos y comparables para todas las regiones, explícitamente vinculados a los objetivos de planificación.

Como ha señalado Wong (2008), la experiencia previa sugiere que no se trata de una tarea fácil. Sin embargo, el desarrollo de un sistema de indicadores más homogéneo facilitaría la comparativa entre distintos ámbitos territoriales. Así mismo, la agregación de los indicadores mediante la aplicación de distintas técnicas (ej. técnicas multicriterio) también es recomendable que se implante a futuro con el objetivo de tener una cuantificación global de la sostenibilidad a nivel regional y subregional. El uso de un índice global subregional permitiría además diferenciar aquellos ámbitos que presentan mejores condiciones de sostenibilidad de aquellos otros más problemáticos en los que es más urgente actuar.

Por otro lado, en lo sucesivo es recomendable que los procesos de evaluación y seguimiento territorial mediante indicadores se coordinen con los de evaluación y seguimiento ambiental. Para ello pueden aplicarse indicadores comunes, que sean útiles en ambos tipos de planificación de cara a sumar sinergias y optimizar los recursos disponibles.

En todo caso, parece conveniente que se sigan potenciando sistemas de evaluación y seguimiento que incluyan el uso de indicadores. Estos sistemas deben permitir evaluar, de forma pragmática, tanto el cumplimiento de las medidas derivadas de los propios planes o estrategias territoriales, como la evolución, en términos de sostenibilidad, del ámbito de actuación e incluso si los instrumentos están cumpliendo su papel como marco de referencia para la multitud de agentes implicados en la ordenación territorial. El uso de indicadores tiene la ventaja de permitir cuantificar estos procesos, ayudar a la comparación entre distintos ámbitos territoriales, facilitar una toma de decisiones más firmemente basada en la evidencia y colaborar en la comunicación de resultados.

Agradecimientos: Este artículo se enmarca dentro de los proyectos de investigación desarrollados entre 2015 y 2017 para la elaboración del Plan Regional de Ordenación Territorial de Cantabria, financiados por la Dirección General de Ordenación del Territorio y Evaluación Ambiental Urbanística del Gobierno de Cantabria.

Declaración responsable: Las/os autoras/es declaran que no existe ningún conflicto de interés con relación a la publicación de este artículo. Asimismo, declaran que han elaborado conjuntamente todos los apartados del artículo, si bien la responsabilidad o principal contribución de cada uno se detalla a continuación. Rubén Cordera: recopilación e interpretación de la información, elaboración de cartografía, análisis de los resultados y redacción del manuscrito; 
Soledad Nogués: planteamiento de la estructura y metodología, redacción y revisión del manuscrito; y Esther González-González: análisis de la información, redacción y revisión del manuscrito. 


\section{Bibliografía}

Ambiente Italia (2003). European Common Indicators. Final Project Report: Development, Refinement, Management and Evaluation of European Common Indicators Project (ECI). Milan: Ambiente Italia Research Institute.

Benach, N., \& Walliser, A. (2011). Introduction to the special issue: urban problems and issues in contemporary Spanish and Portuguese cities. Urban Research \& Practice, 4, 227231. http://dx.doi.org/10.1080/17535069.2011.616742

Bielza de Ory, V. (2010). La ordenación del territorio en Aragón: Enfoques e Instrumentos. Cuadernos Geográficos, 47(2), Retrieved from http://revistaseug.ugr.es/index.php/cuadgeo/article/view/612

BOE (2011). Decreto 19/2011, de 10 de febrero, por el que se aprueban definitivamente las directrices de ordenación del territorio. Boletín Oficial del Estado, num. 46, de 23 de febrero de 2011, pp. 20995-21037.

Brueckner, J. K. (2000). Urban Sprawl: Diagnosis and Remedies. International Regional Science Review, 23, 160-171. http://dx.doi.org/10.1177/016001700761012710

Burriel, E. (2008). La década prodigiosa del urbanismo español (1997-2006). Scripta Nova, XII (270: 64). Retrieved from http://www.ub.es/geocrit/sn/sn-270/sn-270-64.htm

Calderón, B. \& \& García, J. L. (2017). Legislación urbanística y planeamiento urbano en España, 1998-2015. Del despilfarro a la sostenibilidad. Scripta Nova, XXI(570). http://dx.doi.org/10.1344/sn2017.21.19429

Comisión Europea (1999). Estrategia Territorial Europea. Hacia un desarrollo equilibrado y sostenible del territorio de la UE. Postdam: Oficina de Publicaciones de las Comunidades Europeas. Retrieved from http://ec.europa.eu/regional_policy/sources/docoffic/official/reports/pdf/sum_es.pdf Consejo de Europa (1983). Carta europea de ordenación del territorio. Torremolinos: Consejo de Europa. Retrieved from http://docplayer.es/12789917-Carta-europea-de-ordenacion-delterritorio.html

DCLG (2008). Regional Spatial Strategy and Local Development Framework: Core Output Indicators - Update 2/2008. London: Department for Communities and Local Government Retrieved from

http://webarchive.nationalarchives.gov.uk/20120920011524/http://www.communities.gov.uk/ documents/planningandbuilding/pdf/coreoutputindicators2.pdf 
De Vries, A. (2009). Gestión de información territorial para la toma de decisiones. In J. Farinós Dasí (Ed.), Cohesión e inteligencia territorial: Dinámicas y procesos para una mejor planificación y toma de decisiones (pp. 139-152). Valencia: Servei de Publicacions.

Elorrieta, B., Olcina, J., \& Sánchez, D. (2016). La sostenibilidad en la planificación territorial de escala regional en España: estudio de casos. Cuadernos Geográficos, 55, 149-175. Retrieved from http://www.redalyc.org/pdf/171/17146265006.pdf

European Parliament and of the Council (2001). Directive 2001/42/EC of 27 June 2001 on the assessment of the effects of certain plans and programmes on the environment. Official Journal $L$ 197, 30-37. Retrieved from

https://eur-lex.europa.eu/legal-content/EN/ALL/?uri=CELEX:32001L0042

Eurostat [Statistical Office of the European Communities] (2001). Measuring Progress Towards a More Sustainable Europe. Luxembourg: Eurostat. Retrieved from hittps://ec.europa.eu/eurostat/documents/3217494/5619864/KS-77-07-115EN.PDF/06ee41ca-2717-46ee-bee5-07e6bf6c26a2

Ewing, R. (1997). Is Los Angeles-Style Sprawl Desirable? Journal of the American Planning Association, 63, 107-126. http://dx.doi.org/10.1080/01944369708975728

Ewing, R., \& Hamidi, S. (2015). Compactness versus Sprawl: A Review of Recent Evidence from the United States. Journal of Planning Literature, 30, 413432. http://dx.doi.org/10.1177/0885412215595439

Faludi, A. (2000). The performance of spatial planning. Planning practice and Research, 15, 299318. http://dx.doi.org/10.1080/713691907

Faludi, A., \& Waterhout, B. (2006). Introducing evidence-based planning. disP-The Planning Review, 42(165), 4-13.

Farinós, J., Romero, J., \& Sánchez, I. (2005). Structural problems for the renewal of planning styles: the Spanish case. European Planning Studies, 13, 217235. http://dx.doi.org/10.1080/0965431042000321794

Farinós, J. (2008). Gobernanza territorial para el desarrollo sostenible: estado de la cuestión y agenda. Boletín de la Asociación de Geógrafos Españoles, 46, 1132. http://dx.doi.org/10.21138/bage.668

Farinós, J. (2011). De la evaluación ambiental estratégica a la evaluación de impacto territorial: reflexiones acerca de la tarea de evaluación (From strategic environmental assessment to territorial impact assessment: reflections about evaluation practice). Valencia: Universitat de València, Servei de Publicacions. 
Farinós, J. (2014). Evaluación de políticas y dación de cuentas, requisitos para una adecuada gobernabilidad territorial a partir de una nueva planificación; combinando política y geografía. Perspectiva Geográfica, 19(2), 331-356.

Feria Toribio, J. M., Rubio Tenor, M., \& Santiago Ramos, J. (2005). Los planes de ordenación del territorio como instrumentos de cooperación. Boletín de la Asociación de Geógrafos Españoles, 39, 87-116. http://dx.doi.org/10.21138/bage.500

García Martínez, M. (2011). El proceso de Evaluación Territorial, algo más que seguimiento. In J. Farinós Dasí (Ed.), De la evaluación ambiental estratégica a la evaluación de impacto territorial: reflexiones acerca de la tarea de evaluación (From strategic environmental assessment to territorial impact assessment: reflections about evaluation practice) (pp. 331-348). Valencia: Universitat de València, Servei de Publicacions.

Generalitat Valenciana (2015). Libro Digital. Estrategia Territorial de la Comunitat Valenciana. Retrieved from http://www.habitatge.gva.es/es/web/planificacion-territorial-e-infraestructuraverde/libro-digital-77497

Giannakourou, G. (2005). Transforming spatial planning policy in Mediterranean countries: Europeanization and domestic change. European Planning Studies, 13, 319331. http://dx.doi.org/10.1080/0365431042000321857

Gobierno de Aragón (2014a). Estrategia de Ordenación Territorial de Aragón. Modelo Territorial. Zaragoza: Departamento de Política Territorial e Interior. Retrieved from http://www.aragon.es/DepartamentosOrganismosPublicos/Departamentos/VertebracionTerri torioMovilidadVivienda/AreasTematicas/OrdenacionTerritorial/EOTA/

Gobierno de Aragón (2014b) Estrategia de Ordenación Territorial de Aragón. Modelo Territorial. Tomo II. Indicadores. Departamento de Política Territorial e Interior. Retrieved from http://www.aragon.es/estaticos/GobiernoAragon/Departamentos/VertebracionTerritorioMo vilidadTransporte/Areas/01_Ordenacion_territorio/Indicadores.pdf

Gobierno de Navarra (2003). Estrategia Territorial de Navarra: Directrices para la ordenación del territorio. Pamplona: Dirección General de Ordenación del Territorio y Vivienda del Gobierno de Navarra. Retrieved from http://www.nasuvinsa.es/sites/default/files/pdfs/ETN_000.pdf

Gobierno Vasco (2016). Revisión de las Directrices de Ordenación Territorial de la Comunidad Autónoma del País Vasco. Vitoria-Gasteiz: Departamento de Medio Ambiente y Política Territorial. Retrieved from

http://www.euskadi.eus/web01-

a2lurral/es/contenidos/informacion/revision_dot/es_def/index.shtml 
Gobierno Vasco (2017a). Indicadores territoriales. Departamento de Medio ambiente, Planificación Territorial y Vivienda. Retrieved from http://www.ingurumena.ejgv.euskadi.eus/r49aa33a/es/aa33alndicadoresWAR/indicadores/SP/index.jsp

Gobierno Vasco (2017b). Indicadores de sostenibilidad territorial y urbanística. Departamento de Desarrollo Económico e Infraestructuras. Retrieved from

http://www.euskadi.eus/informacion/indicadores-de-sosteniblidad-territorial-y-urbanistica090300/web01-a2estadi/es/

González, J. L., \& Martínez, R. (1980). Ordenación territorial en una nueva situación. Ciudad y Territorio, 1, 25-38.

Guyadeen, D., \& Seasons, M. (2018). Evaluation Theory and Practice: Comparing Program Evaluation and Evaluation in Planning. Journal of Planning Education and Research, 38(1), 98110. http://dx.doi.org/10.1177/0739456X16675930

Hildenbrand, A. (2006). La política de ordenación del territorio de la Comunidades Autónomas: balance crítico y propuestas para la mejora de su eficacia. Revista de derecho urbanístico y medio ambiente, 230, 79-140.

Juaristi, J. (2009). La Ordenación del Territorio en el umbral del año 2010: promesas, retos y problemas. Lurralde: Investigación y espacio, 32, 361-382. Retrieved from https://dialnet.unirioja.es/servlet/articulo?codigo=3179541

Laurian, L., Crawford, J., Day, M., Kouwenhoven, P., Mason, G., Ericksen, N., \& Beattie, L. (2010). Evaluating the Outcomes of Plans: Theory, Practice, and Methodology. Environment and Planning B: Planning and Design, 37(4), 740-757. http://dx.doi.org/10.1068/b35051

Laurian, L., Day, M., Berke, P., Ericksen, N., Backhurst, M., Crawford, J., \& Dixon, J. (2004). Evaluating Plan Implementation: A Conformance-Based Methodology. Journal of the American Planning Association, 70(4), 471-480. https://doi.org/10.1080/01944360408976395

Lois González, R.C., \& Aldrey Vázquez, J.A. (2011) El problemático recorrido de la ordenación del territorio en Galicia. Cuadernos Geográficos, 47(2010-2), 583-610. Retrieved from http://revistaseug.ugr.es/index.php/cuadgeo/article/view/620/708

Lozano Valencia, P. J. (2004). La ordenación territorial en el País Vasco. Debilidades, amenazas, fortalezas y oportunidades de la escala intermedia. Investigaciones Geográficas, 32, 157-176. Retrieved from https://www.investigacionesgeograficas.com/article/view/2003-n32-laordenacion-territorial-en-el-pais-vasco-debilidades-amenazas-fortalezas-y-oportunidades-de-la-escalaintermedia 
Lozano Valencia, P. J., Latas Zaballos, I., \& Ruiz Vaqueriza, A. (2015). Los procesos de gobernanza territorial dentro de la ordenación del territorio del País Vasco. Evaluación del grado de eficiencia. In J. de la Riva, P. Ibarra, R. Montorio \& M. Rodrigues (Eds.), Análisis espacial y representación geográfica: innovación y aplicación (pp. 235-244). Universidad de Zaragoza.

Lunt, N., Davidson, C., \& McKegg, K. (2003). Evaluating Policy and Practice. Auckland: Pearson Education.

Mascarenhas, A., Ramos, T. B., \& Nunes, L. (2012). Developing an integrated approach for the strategic monitoring of regional spatial plans. Land Use Policy, 29(3), 641-651. doi:https://doi.org/10.1016/j.landusepol.2011.10.006

Ministerio de Medio Ambiente (2008). Instrumentos de Ordenación Territorial. Madrid: Ministerio de Medio Ambiente. Retrieved from http://www.mapama.gob.es/es/desarrollo-rural/planes-yestrategias/informacion-territorial/instrumentos-de-ordenacion-territorial/

Nasuvinsa (2017) ETN. Informes de vigencias y memorias. Retrieved from http://etnnasuvinsa.opendata.arcgis.com/

Nogués, S. (1992). La comarca en la planificación y gestión del territorio. Santander: Departamento de Geografía, Urbanismo y Ordenación del Territorio. Universidad de Cantabria.

ODPM (2002) Monitoring Regional Planning Guidance: Good Practice Guidance on Targets and Indicators. London: Office of the Deputy Prime Minister.

Oliveira, V., \& Pinho, P. (2010). Measuring success in planning: Developing and testing a methodology for planning evaluation. Town Planning Review, 81(3), 307-332.

OTN (2016) Seguimiento anual 2015: resumen ejecutivo. Estrategia Territorial de Navarra. Observatorio Territorial de Navarra. Retrieved from

http://www.nasuvinsa.es/sites/default/files/pdfs/160302_ETN_SeguimientoAnual2015_v5_.pdf Ponce, J. (2004). Land use law, liberalization, and social cohesion through affordable housing in Europe: the Spanish case. The urban lawyer, 36, 317-340. Retrieved from https://www.jstor.org/stable/27895489

Rogers, P. J., Petrosino, A., Huebner, T. A., \& Hacsi, T. A. (2000). Program theory evaluation: Practice, promise, and problems. New directions for evaluation, 2000(87), 5-13.

Segura, S., \& Pedregal, B. (2017). Monitoring and Evaluation Framework for Spatial Plans: A Spanish Case Study. Sustainability, 9(10), 1706. http://dx.doi.org/10.3390/su9101706

Seixas, J., \& Albet, A. (2016). Urban Governance in Southern Europe. Abingdon: Routledge. 
Tanguay, G. A., Rajaonson, J., Lefebvre, J. F., \& Lanoie, P. (2010). Measuring the sustainability of cities: An analysis of the use of local indicators. Ecological Indicators, 10, 407418. http://dx.doi.org/10.1016/j.ecolind.2009.07.013

Terán, F. (1982). Planeamiento urbano en la España contemporánea (1900-1980). Madrid: Alianza.

UNCED [United Nations Commission on Environment and Development] (1992). United Nations conference on Environment \& Development. Rio de Janeiro, Brazil, June 3-14. Agenda 21. Retrieved from https://sustainabledevelopment.un.org/outcomedocuments/agenda21

UNCSD [United Nations Commission on Sustainable Development] (1996). Indicators of Sustainable Development: Framework and Methodologies. New York: UNCSD. Retrieved from http://www.un.org/esa/sustdev/csd/csd9_indi_bp3.pdf

UNDP [United Nations Development Programme. Evaluation Office] (2002). Handbook on monitoring and evaluating for results. Evaluation Office.

Valenzuela, L. M., \& Soria, J. A. (2011). Observatorios territoriales y urbanos en Europa ¿̇entidades pasivas 0 instrumentos operativos para la planificación? Ciudad y territorio: estudios territoriales, $168,243-260$.

Wong, C. (2006). Indicators for urban and regional planning: the interplay of policy and methods. Abingdon: Routledge.

Wong, C., Rae, A., Baker, M., Hincks, S., Kingston, R., Watkins, C., \& Ferrari, E. (Eds.) (2008). Measuring the Outcomes of Spatial Planning in England. London: Royal Town Planning Institute and the Department for Communities and Local Government.

Xunta de Galicia (2011). Directrices de Ordenación do Territorio de Galicia e da Sustentabilidade Territorial (PSST). Santiago de Compostela: Consellería de Medio Ambiente e Ordenación do Territorio. Retrieved from https://cmaot.xunta.gal/seccionorganizacion/c/CMAOT_Instituto_Estudos_Territorio?content=Direccion_Xeral_Sostibilidade_Paisax e/Directrices_Ordenacion_Territorio/seccion.html\&std=descarga-dot.html

Xunta de Galicia (2013). Plan do Seguimento das Directrices de Ordenación do Territorio de Galicia e da Sustentabilidade Territorial (PSST). Santiago de Compostela: Consellería de Medio Ambiente e Ordenación do Territorio. Retrieved from http://cmaot.xunta.gal/seccionorganizacion/c/Instituto_Estudos_Territorio?content=Direccion_Xeral_Sostibilidade_Paisaxe/Seguim ento_DOT/seccion.html\&sub=Plan_seguimento_DOT_PSST/ 
Xunta de Galicia (2018). Informes PSST. Retrieved from https://cmaot.xunta.gal/seccionorganizacion/c/Instituto_Estudos_Territorio?content=Direccion_Xeral_Sostibilidade_Paisaxe/Seguim ento_DOT/seccion.html\&sub=Informes_PSST/\#

Zarraluqui, L. (2003). La Estrategia Territorial de Navarra, primera experiencia en España de aplicación de los principios de planificación y desarrollo espacial europeos a un nivel regional. Urban, 8, 111-122. Retrieved from http://polired.upm.es/index.php/urban/article/view/399 